\title{
LHCb results on exotics and pentaquark states
}

\author{
Marco Pappagallo ${ }^{1, \star}$ \\ ${ }^{1}$ INFN and Universitá degli studi di Bari
}

\begin{abstract}
The latest years have seen a resurrection of interest in searches for exotic states. Using the data collected at pp collisions at 7 and $8 \mathrm{TeV}$ by the LHCb experiment, unambiguous new observations of exotic charmonia hadrons produced in $B$ and $\Lambda_{b}$ decays are presented. Results of a search for a tetraquark state decaying into $B_{s}^{0} \pi^{ \pm}$arealso reported.
\end{abstract}

\section{Introduction}

The LHCb detector [1] is a single-arm forward spectrometer covering the pseudorapidity range $2<\eta<5$, designed for the study of particles containing $b$ or $c$ quarks. During 2011 and 2012, pp collision data corresponding to integrated luminosities of $1 \mathrm{fb}^{-1}$ at a centre-of-mass energy of $7 \mathrm{TeV}$ and $2 \mathrm{fb}^{-1}$ at $8 \mathrm{TeV}$ have been collected with the $\mathrm{LHCb}$ detector.

\section{Search for pentaquarks states}

\subsection{Observation of $J / \psi p$ resonances consistent with pentaquark states in $\Lambda_{b} \rightarrow J / \psi K^{-} p$ decays}

Past claimed observations of pentaquark states have been shown to be spurious but the existence of pentaquark baryon states would not be surprising given the observation of charged charmonium-like meson (e.g. $\left.Z_{c}(4430)^{+}\right)$. A large sample (26,000 candidates) of $\Lambda_{b}$ baryons decaying to the $J / \psi K^{-} p$ final state has been reconstructed at LHCb [2]. In Fig. 1(a) the Dalitz plot is shown using the $K^{-} p$ and $J / \psi p$ invariant masses-squared as independent variables. A distinct horizontal band is observed in the $J / \psi p$ invariant mass distribution near $19.5 \mathrm{GeV} / c^{2}$. In order to ascertain if the observed structures are resonant in nature and not due to reflections generated by the $\Lambda^{*}$ states, it is necessary to perform a full amplitude analysis, allowing for interference effects between both decay sequences.

The fit uses five decay angles and the $K^{-} p$ invariant mass $m_{K p}$ as independent variables. First the data were fit with an amplitude model that contains $14 \Lambda^{*}$ states listed by the Particle Data Group [3]. As this did not give a satisfactory description of the data, one $P_{c}^{+}$state was added, and when that was not sufficient a second state was included. The two $P_{c}^{+}$states are found to have masses of $4380 \pm$ $8 \pm 29 \mathrm{MeV}$ and $4449.8 \pm 1.7 \pm 2.5 \mathrm{MeV}$, with corresponding widths of $205 \pm 18 \pm 86 \mathrm{MeV}$ and $39 \pm 5 \pm 19 \mathrm{MeV}$. The best fit solution has spin-parity $J^{P}$ values of $\left(3 / 2^{-}, 5 / 2^{+}\right)$. Acceptable solutions are also found for additional cases with opposite parity, either $\left(3 / 2^{+}, 5 / 2^{-}\right)$or $\left(5 / 2^{+}, 3 / 2^{-}\right)$. The best fit projections are shown in Fig. 1(b). The significances of the lower mass and higher mass states are 9 and 12 standard deviations, respectively.

\footnotetext{
^e-mail: marco.pappagallo@cern.ch
} 

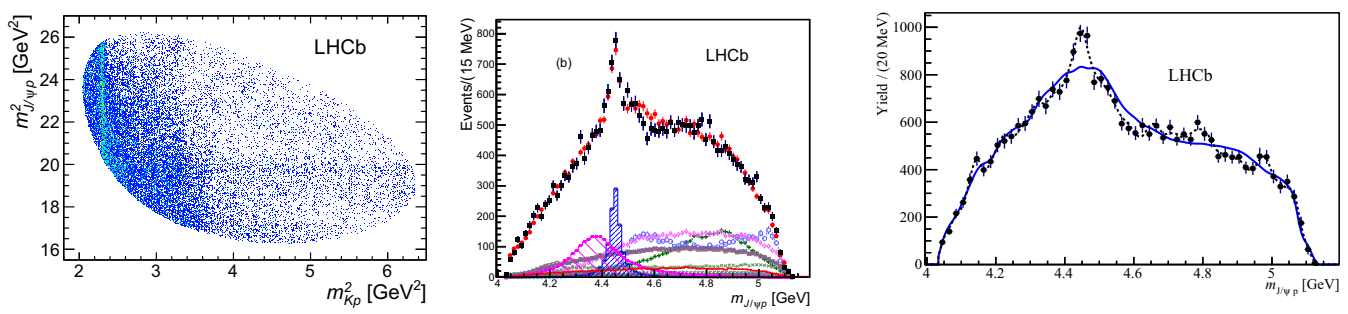

Figure 1. (left) Invariant mass squared of $K^{-} p$ versus $J / \psi p$ for candidates. (middle) Fit projections for $m_{J / \psi p}$ with two $P_{c}^{+}$states. The data are shown as solid (black) squares, while the solid (red) points show the results of the fit. The (blue) open squares with the shaded histogram represent the $P_{c}(4450)^{+}$state, and the shaded histogram topped with (purple) filled squares represents the $P_{c}(4380)^{+}$state. (right) Efficiency-corrected and backgroundsubtracted $m_{J / \psi p}$ distribution of the data (black points), with $P D F\left(m_{J / \psi p} \mid H_{0}\right)$ (solid blue line) and $P D F\left(m_{J / \psi p} \mid H_{1}\right)$ (dashed black line) superimposed.

Further evidence for the resonant character of the higher mass, narrower, $P_{c}^{+}$state is obtained by viewing the evolution of the complex amplitude in the Argand diagram [3]. In the amplitude fits discussed above, the $P_{c}(4450)^{+}$is represented by a Breit-Wigner amplitude, where the magnitude and phase vary with $m_{J / \psi p}$ according to an approximately circular trajectory in the $\left(\operatorname{Re} A^{P_{c}}, \operatorname{Im} A^{P_{c}}\right.$ ) plane, where $A^{P_{c}}$ is the $m_{J / \psi p}$ dependent part of the $P_{c}(4450)^{+}$amplitude. An additional fit to the data has been performed, in which the $P_{c}(4450)^{+}$amplitude is represented as the combination of independent complex amplitudes at six equidistant points in the range $\pm \Gamma_{0}=39 \mathrm{MeV}$ around $M_{0}=4449.8 \mathrm{MeV}$ as determined in the default fit. The resulting Argand diagram, shown in Fig. 2(a), is consistent with a rapid counter-clockwise change of the $P_{c}(4450)^{+}$phase when its magnitude reaches the maximum, a behavior characteristic of a resonance. A similar study for the wider state is shown in Fig. 2(b); although the fit does show a large phase change, the amplitude values are sensitive to the details of the $\Lambda^{*}$ model and so this latter study is not conclusive.

\subsection{Model-independent evidence for $J / \psi p$ contributions to $\Lambda_{b} \rightarrow J / \psi p K^{-}$decays}

In order to investigate further the presence of pentaquarks in the $\Lambda_{b} \rightarrow J / \psi p K^{-}$decay chain, a modelindependent analysis has been carried out [4]. It has been demonstrated at more than 9 standard deviations that the $\Lambda_{b} \rightarrow J / \psi p K^{-}$decays cannot all be attributed to $K^{-} p$ resonant or nonresonant contributions (Fig. 1(c)). The analysis requires only minimal assumptions on the mass and spin of the $K^{-} p$ contributions; no assumptions on their number, their resonant or nonresonant nature, or their lineshapes have been made. Non- $K^{-} p$ contributions, which must be present in the data, can be either of the exotic hadron type, or due to rescattering effects among ordinary hadrons. This result supports the amplitude model-dependent observation of the $J / \psi p$ resonances presented previously [2].

\subsection{Evidence for exotic hadron contributions to $\Lambda_{b} \rightarrow J / \psi p \pi^{-}$decays}

Observations of the same two pentaquark states in another decay would strengthen their interpretation as genuine exotic baryonic states, rather than kinematical effects related to the so called triangle singularity [5,6]. The LHCb collaboration has performed an amplitude analysis of $\Lambda_{b} \rightarrow J / \psi p \pi^{-}$ decays, which are related to $\Lambda_{b} \rightarrow J / \psi p K^{-}$decays via Cabibbo suppression [7]. A sample of $1885 \pm$ 50 candidates has been selected using the same data sample $\left(3 \mathrm{fb}^{-1}\right)$ and similar seletion tecnique as 


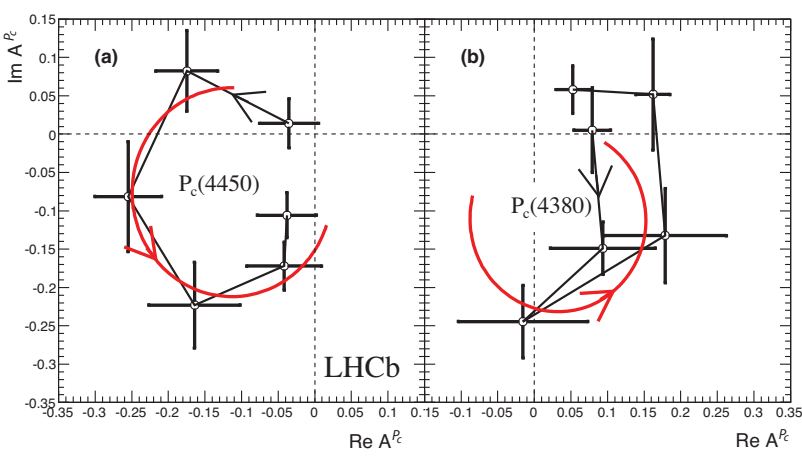

Figure 2. Fitted values of the real and imaginary parts of the amplitudes for the baseline $\left(3 / 2^{-}, 5 / 2^{+}\right)$fit for a) the $P_{c}(4450)^{+}$state and b) the $P_{c}(4380)^{+}$state, each divided into six $m_{J / \psi p}$ bins of equal width between $-\Gamma_{0}$ and $+\Gamma_{0}$ shown in the Argand diagrams as connected points with error bars.

for the $\Lambda_{b} \rightarrow J / \psi p K^{-}$decays. Amplitude models for the $\Lambda_{b} \rightarrow J / \psi p \pi^{-}$decays are constructed to examine the possibility of exotic hadron contributions from the $P_{c}(4380)^{+}$and $P_{c}(4450)^{+} \rightarrow J / \psi p$ states [2] and from the $Z_{c}(4200)^{-} \rightarrow J / \psi \pi^{-}$state, previously reported by the Belle collaboration in $B^{0} \rightarrow J / \psi K^{+} \pi^{-}$decays [8].

The 15 well-established $N^{*}$ resonances [3] are considered in the amplitude model of $p \pi^{-}$contributions. The high-mass and high-spin states are not included, since they require $L \geq 3$ in the $\Lambda_{b}$ decay and therefore are unlikely to be produced near the upper kinematic limit of $m_{p \pi}$. The data are examined only for the presence of the previously observed $P_{c}(4380)^{+}, P_{c}(4450)^{+}$states and the claimed $Z_{c}(4200)^{-}$resonance due to the limited number of signal events and the large number of free parameters. In the fits, the mass and width of each exotic state are fixed to the reported central values.

A significantly better description of the data is achieved by either including the two $P_{c}^{+}$states observed in $\Lambda_{b} \rightarrow J / \psi p K^{-}$decays, or the $Z_{c}(4200)^{-}$state (Fig. 3(a)). If both types of exotic resonances are included, the total significance for them is $3.1 \sigma$. Individual exotic hadron components, or the two $P_{c}^{+}$states taken together, are not significant as long as the other(s) is (are) present. Within the statistical and systematic errors, the data are consistent with the $P_{c}(4380)^{+}$and $P_{c}(4450)^{+}$production rates expected from their previous observation and Cabibbo suppression.

\section{Amplitude analysis of $B^{+} \rightarrow J / \psi \phi K^{+}$and observation of $J / \psi \phi$ structures consistent with exotic states}

There has been a great deal of experimental and theoretical interest in $J / \psi \phi$ mass structures in $B^{+} \rightarrow$ $J / \psi \phi K^{+}$decays since the CDF collaboration presented $3.8 \sigma$ evidence for a near-threshold $X(4140)$ mass peak, with width $\Gamma=12 \mathrm{MeV}$ [9]. Much larger widths are expected for charmonium states at this mass because of open flavor decay channels, which should also make the kinematically suppressed $X \rightarrow J / \psi \phi$ decays undetectable. Therefore, it has been suggested that the $X(4140)$ peak could be a molecular state, a tetraquark state, a hybrid state, or a rescattering effect.

The LHCb collaboration has performed the first amplitude analysis of $B^{+} \rightarrow J / \psi \phi K^{+}$decays $[10,11]$. We have obtained a good description of the data in the $6 \mathrm{D}$ phase space composed of invariant masses and decay angles (Fig. 3(b)). The $K^{*+}$ amplitude model extracted from our data is consistent with expectations from the quark model and from the previous experimental results on such 

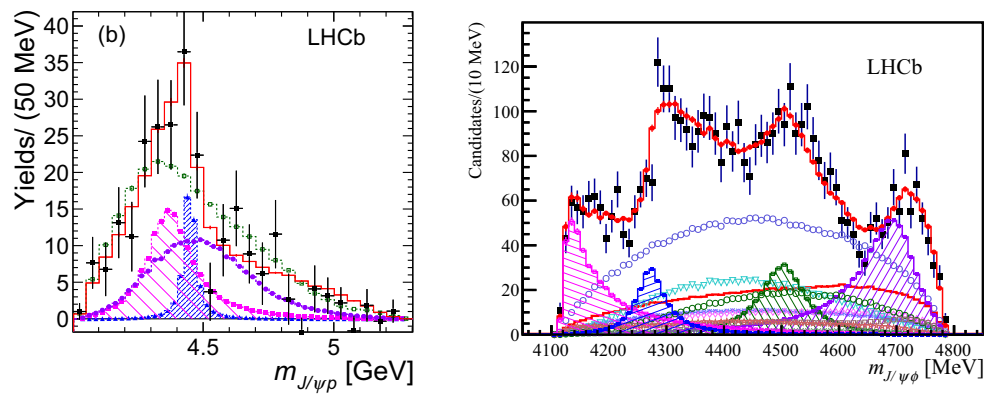

Figure 3. (left) Background-subtracted data and fit projections onto $m(J / \psi p)$ for the $m(p \pi)>1.8 \mathrm{GeV}$ region. (right) Distributions of $J / \psi \phi$ invariant masses for the $B^{+} \rightarrow J / \psi \phi K^{+}$candidates (black data points) compared with the results of the default amplitude fit containing eight $K^{*+} \rightarrow \phi K^{+}$and five $X \rightarrow J / \psi \phi$ contributions. The total fit is given by the red points with error bars.

resonances. We determine the $J^{P C}$ quantum numbers of the $X(4140)$ structure to be $1^{++}$. This has a large impact on its possible interpretations, in particular ruling out the $0^{++}$or $2^{++} D_{s}^{*+} D_{s}^{*-}$ molecular models. The $X(4140)$ width is substantially larger than previously determined. The existence of the $X(4274)$ structure is established and its quantum numbers are determined to be $1^{++}$. The high $J / \psi \phi$ mass region is investigated for the first time with good sensitivity and shows very significant structures, which can be described as two $0^{++}$resonances: $X(4500)$ and $X(4700)$.

\section{Search for structure in the $B_{s}^{0} \pi^{ \pm}$invariant mass spectrum}

In february the D0 collaboration has claimed [12] the observation with $5.1 \sigma$ significance of a narrow structure, which they dub the $X(5568)$, in the decay sequence $X(5568) \rightarrow B_{s}^{0} \pi^{ \pm}, B_{s}^{0} \rightarrow J / \psi \phi$, $J / \psi \rightarrow \mu^{+} \mu^{-}, \phi \rightarrow K^{+} K^{-}$. The mass and width of the $X(5568)$ state are reported to be $m=$ $5567.8 \pm 2.9_{-1.9}^{+0.9} \mathrm{MeV} / \mathrm{c}^{2}$ and $\Gamma=21.9 \pm 6.4_{-2.5}^{+5.0} \mathrm{MeV} / \mathrm{c}^{2}$. The fraction of $B_{s}^{0}$ mesons that comes from $X(5568) \rightarrow B_{s}^{0} \pi^{ \pm}$decay is reported to be $\rho_{X}^{\mathrm{DO}}=(8.6 \pm 1.9 \pm 1.4) \%$. Recently the significance of the signal has been revisited after loosening the selection of the $B_{s}^{0} \pi^{ \pm}$candidates.

The claimed $X(5568)$ state has been searched in a data sample corresponding to $3 \mathrm{fb}^{-1}$ of $p p$ collision data at $\sqrt{s}=7$ and $8 \mathrm{TeV}$ recorded by LHCb [13]. The $B_{s}^{0}$ mesons are reconstructed in decays to $D_{s}^{-} \pi^{+}$and $J / \psi \phi$ final states to obtain a $B_{s}^{0}$ yield approximately 20 times larger than that available to the D0 collaboration. The analysis techniques follow closely those developed for studies of the $B^{+} K^{-}$[14], $B^{+} \pi^{-}$and $B^{0} \pi^{+}[15]$ spectra. The $B_{s}^{0}$ candidates are combined with each track that originates from the same primary vertex and that has $p_{T}>500 \mathrm{MeV} / \mathrm{c}$.

The $Q \equiv m\left(B_{s}^{0} \pi\right)-M_{B_{s}^{0}}-M_{\pi}$ distributions are fitted with a function containing components for signal and background (Fig. 4(a)). The signal shape is an S-wave Breit-Wigner function, with mass and width parameters fixed according to the central values obtained by the D0 collaboration. The background is modelled with a polynomial function. The fit has a p-value of $34.0 \%$ and no significant $X(5568)$ yield.

The results of the fits to the $B_{s}^{0} \pi^{ \pm} Q$ value distributions are used to measure the ratio of crosssections

$$
\rho_{X}^{\mathrm{LHCb}} \equiv \frac{\sigma(p p \rightarrow X(5568)+\text { anything }) \times \mathcal{B}\left(X(5568) \rightarrow B_{s}^{0} \pi^{ \pm}\right)}{\sigma\left(p p \rightarrow B_{s}^{0}+\text { anything }\right)}=\frac{N(X)}{N\left(B_{s}^{0}\right)} \times \frac{1}{\epsilon^{\mathrm{rel}}(X)},
$$



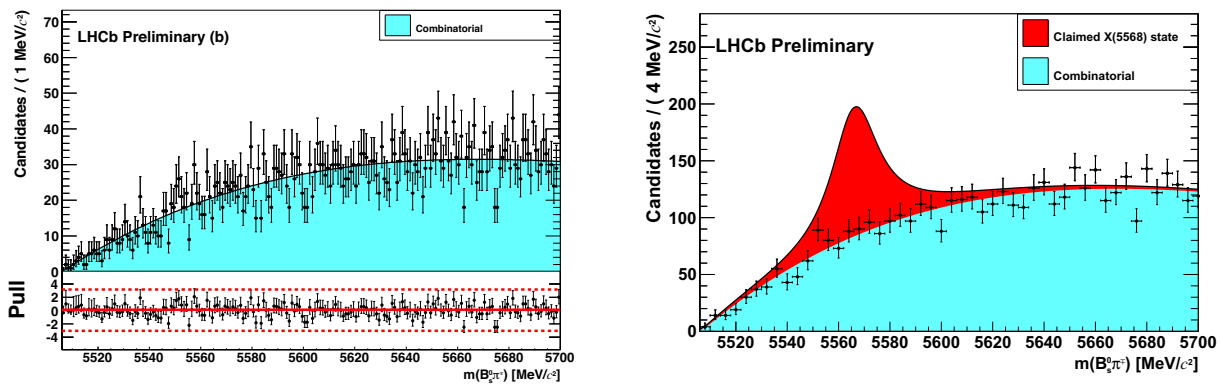

Figure 4. (left) Results of the fit to the $Q$ value distribution, shifted to display the $B_{s}^{0} \pi^{+}$invariant mass, for $B_{s}^{0} \pi$ candidates (both $B_{s}^{0}$ modes combined) with the signal yield fixed to zero. The fits are shown for minimum $B_{s}^{0}$ $p_{T}$ of $10 \mathrm{GeV} / \mathrm{c}^{2}$. (right) Distribution of $Q$ value, shifted to display the $B_{s}^{0} \pi^{+}$invariant mass, for candidates with minimum $B_{s}^{0} p_{T}$ of $10 \mathrm{GeV} / c^{2}$, with a signal component superimposed that corresponds to $\rho_{X}^{\mathrm{LHCb}}=8.6 \%$.

where the cross-sections $\sigma$ are for promptly produced particles within the LHCb acceptance. The relative efficiency $\epsilon^{\mathrm{rel}}(X)=\frac{\epsilon(X)}{\epsilon\left(B_{s}^{0}\right)}$ is determined from simulation and accounts for the reconstruction and selection efficiency of the companion pion as well as the requirement that is within the LHCb acceptance. The dominant sources of systematic uncertainties are due to the variation of the signal width and the effect of the efficiency on the signal shape. Since the signal is not significant, upper limits on $\rho_{X}^{\mathrm{LHCb}}$ are found to be

$$
\begin{aligned}
& \rho_{X}^{\mathrm{LHCb}}\left(B_{s}^{0} p_{T}>5 \mathrm{GeV} / \mathrm{c}^{2}\right)<0.009(0.010) @ 90(95) \% \mathrm{CL}, \\
& \rho_{X}^{\mathrm{LHCb}}\left(B_{s}^{0} p_{T}>10 \mathrm{GeV} / \mathrm{c}^{2}\right)<0.016(0.018) @ 90(95) \% \mathrm{CL} .
\end{aligned}
$$

\section{References}

[1] A. A. Alves Jr. et al. [LHCb Collaboration], JINST 3 (2008) S08005.

[2] R. Aaij et al. [LHCb Collaboration], Phys. Rev. Lett. 115 (2015) 072001.

[3] K. A. Olive et al. (Particle Data Group), Chin. Phys. C, 38 (2014) 090001.

[4] R. Aaij et al. [LHCb Collaboration], Phys. Rev. Lett. 117 (2016) 082002.

[5] T. J. Burns, Eur. Phys. J. A51 (2015) 152.

[6] E. Wang et al., Phys. Rev. D93 (2016) 094001.

[7] R. Aaij et al. [LHCb Collaboration], Phys. Rev. Lett. 117 (2016) 082003.

[8] K. Chilikin et al. [Belle Collaboration], Phys. Rev. D90 (2014) 112009.

[9] T. Aaltonen et al., [CDF collaboration] Phys. Rev. Lett. 102 (2009) 242002.

[10] R. Aaij et al. [LHCb Collaboration], arXiv:1606.07895.

[11] R. Aaij et al. [LHCb Collaboration], arXiv:1606.07898.

[12] V. M. Abazov et al. [D0 Collaboration], Phys. Rev. Lett. 117 (2016) 022003.

[13] R. Aaij et al. [LHCb Collaboration], LHCb-CONF-2016-004 (superseded by Phys. Rev. Lett. 117 (2016) 152003).

[14] R. Aaij et al. [LHCb Collaboration], Phys. Rev. Lett. 110 (2013) 151803.

[15] R. Aaij et al. [LHCb Collaboration], JHEP 1504 (2015) 024. 